\title{
Spin Glasses and Other Lattice Systems with Long Range Interactions
}

\author{
J. Fröhlich and B. Zegarlinski`
}

Theoretical Physics, ETH-Hönggerberg, CH-8093 Zürich, Switzerland

\begin{abstract}
We study classical lattice systems, in particular real spin glasses with Ruderman-Kittel interactions and dipole gases, with interactions of very long (non-summable) range but variable sign. Using the Kac-Siegert representation of such systems and Brascamp-Lieb inequalities we are able to establish detailed properties of the high-temperature phase, such as decay of connected correlations, for these systems.
\end{abstract}

\section{Introduction}

In this paper we study the equilibrium statistical mechanics of classical spin systems with long-range exchange couplings of variable sign. A typical example of a system we propose to consider is a real spin glass with exchange couplings of Ruderman-Kittel (RKKY) type [1]. The Hamiltonian of such a system has the following structure:

$$
H=-\sum_{i, j} \sum_{a, b} J_{i j}^{a b} n_{i} \sigma_{i}^{a} n_{j} \sigma_{j}^{b}-\sum_{i} h_{i}^{3} n_{i} \sigma_{i}^{3} .
$$

Here $i$ and $j$ are sites of a lattice $\Gamma$ (typically chosen to be $\mathbb{Z}^{d}, d=2,3, \ldots$ ); $\sigma_{i}=\left(\sigma_{i}^{1}, \ldots, \sigma_{i}^{N}\right), N=1,2,3, \ldots$, is a classical spin variable at site $i ; n_{i}$ is a random variable taking the values 0 or 1 which indicates whether site $i$ is occupied by a magnetic atom or ion $\left(n_{i}=1\right)$ or by a non-magnetic one $\left(n_{i}=0\right)$. The exchange couplings $J_{i j}^{a b}$ are of long range and can be ferromagnetic or antiferromagnetic. We assume that they are the Fourier transforms of matrix-valued functions on the first Brillouin zone that are bounded in norm. As an example, we shall consider

$$
J_{i j}^{a b}=\delta^{a b} \frac{1}{|i-j|+\lambda}\left(\frac{-k_{F}|i-j| \cos k_{F}|i-j|+\sin k_{F}|i-j|}{k_{F}|i-j|^{3}}\right) .
$$

Such models describe alloys of magnetic atoms or ions in a nonmagnetic host material, e.g. AuFe or CuMn.

\footnotetext{
* Permanent address: Institute of Theoretical Physics, University of Wrocław, Wrocław, Poland
} 
In the study of the statistical properties of such systems one is hampered by the circumstance that

$$
\sum_{j \in \Gamma} \sum_{a=1}^{N}\left|J_{i j}^{a b}\right| \text { diverges. }
$$

This property renders even the analysis of the paramagnetic high-temperature phase rather difficult. Standard high-temperature series diverge at temperatures much higher than a true transition temperature because of the presence of Griffiths singularities [2]. In order to circumvent these difficulties, we shall rewrite spin systems in the Kac representation [3]. In this representation, spin systems become lattice field theories which satisfy Brascamp-Lieb inequalities [4]. It turns out that Brascamp-Lieb inequalities provide a surprisingly powerful tool for the analysis of lattice field theories in the single phase region. (Another related tool that is sometimes available and useful is the Fortuin-Kasteleyn-Ginibre inequalities [5].) We systematically explore these tools and find that they yield detailed information about thermodynamic and correlation functions in the disordered phase of not only spin glasses, but other statistical systems with long-range interactions such as dipole gases.

Unfortunately, our analysis is too soft to provide real insights into properties of the phase diagram at low temperatures. It has recently been proven rigorously that the Sherrington-Kirkpatrick mean-field spin glass models exhibit a genuine phase transition in zero magnetic field, as the temperature is lowered $[6,7]$. There is increasing numerical evidence that short-range Ising spin glasses without external magnetic field exhibit an equilibrium phase transition in dimension three or higher $[8,9]$, and this is supported by analytical, but heuristic arguments [10]. If the exchange couplings in a spin system are of finite range and have a strong ferromagnetic bias, the existence of a phase transition and of spontaneous magnetization at low temperatures can be proven with the help of a Peierls argument. Phase transitions and ordered states at low temperatures in dipole systems with hard-core exclusion have been rigorously exhibited in [12].

But for spin glasses we have no real mathematical understanding of the lowtemperature phase diagram or the system's reaction to a weak external magnetic field. The methods developed in this paper do not appear to enable us to make decisive progress in that direction. They do, however, permit us to study the hightemperature properties in detail and to prove meanfield type upper bounds on transition temperatures.

Our paper is organized as follows. In Sect. 1 we define the class of lattice systems analyzed in this paper, introduce our notations and summarize our main results in a mathematically precise form.

In Sect. 2 we prove that the thermodynamic limit of the pressure of a large class of spin systems in zero magnetic field exists at arbitrary temperatures and is selfaveraging in the randomness. These results are then extended to systems in a nonvanishing external field at sufficiently high temperatures.

In Sect. 3 we convert lattice spin systems to lattice field theories, with the help of the Kac representation. We relate correlation functions of spin systems to correlation functions of equivalent lattice field theories, using "integration-byparts" identities. We then review the Brascamp-Lieb inequalities and show how they apply to our systems. 
In Sect. 4 we use the tools prepared in Sect. 3 to study the decay of connected correlation functions. We prove bounds on a variety of quenched susceptibilities.

In Sect. 5 we consider ergodic averages of correlation functions. We show that, at high temperature, the thermodynamic limits of these quantities exist and are independent of boundary conditions and of the sample of magnetic impurities chosen. This implies, in particular, that, in zero magnetic field, the EdwardsAnderson order parameter vanishes, independent of boundary conditions, if the temperature is large enough.

In an Appendix, we prove some technical results concerning the class of exchange interactions studied in this paper.

It is straightforward to extend our methods and results to other lattice systems with long range interactions such as dipole gases.

\section{Notation and Results}

Let $\mathscr{F}$ be the family of bounded sets in $\mathbb{Z}^{d}$. Let $\mathscr{F}_{0} \equiv\left\{\Lambda_{k} \in \mathscr{F}\right\}_{k \in \mathbb{N}}$ be an increasing sequence - called a countable base of $\mathscr{F}$ - satisfying the following property: For any $\Lambda \in \mathscr{F}$, there is $k \in \mathbb{N}$ s.t. $\Lambda \subset \Lambda_{k^{\prime}}$ for all $k^{\prime} \geqq k$. A countable base is called exponential, and is denoted by $\mathscr{F}_{\text {exp }}$, iff for any $k \in \mathbb{N}$

$$
\Lambda_{k+1}=\bigcup_{l=1, \ldots, L^{d}} \Lambda_{k}^{(l)}
$$

with some $L \in \mathbb{N}, L>1$;

$$
\Lambda_{k}^{(l)} \equiv\left\{i \in \mathbb{Z}^{d}: i-x_{l} \in \Lambda_{k}\right\}, \quad l=1, \ldots, L^{d},
$$

where $x_{l} \in \mathbb{Z}^{d}$ are chosen so that

$$
\Lambda_{k}^{(l)} \cap \Lambda_{k}^{\left(l^{\prime}\right)}=\emptyset \quad \text { if } \quad l \neq l^{\prime} .
$$

The volume, $|\Lambda|$, of some region $\Lambda \in \mathscr{F}$ is, by definition the number of elements in $\Lambda$. By assumptions (1)-(3), the volume of $\Lambda_{k+1} \in \mathscr{F}_{\exp }$ satisfies

$$
\left|\Lambda_{k+1}\right|=L^{d}\left|\Lambda_{k}\right|
$$

If not stated otherwise, a countable base, $\mathscr{F}_{0}$, is assumed to be a van-Hove sequence. We define $S_{N}$ to be equal to the set $\{-1,1\}$, for $N=1$, and to the unit sphere $S^{N-1} \subset \mathbb{R}^{N}$, for $N \geqq 2$. A classical spin at site $i \in \mathbb{Z}^{d}$ is a vector $\sigma_{i} \equiv\left(\sigma_{i}^{a}\right.$ : $a=1, \ldots, N) \in S_{N}$. Our space of spin configurations is $\Omega \equiv\left(S_{N}\right)^{\mathbb{Z}^{d}}$, with elements $\sigma \equiv\left(\sigma_{i}\right)_{i \in \mathbb{Z}^{d}}$. Let $\Sigma$ denote a $\sigma$-algebra of subsets in $\Omega$, generated by the Tychonov topology. For $\Lambda \in \mathscr{F}$, let $\Sigma_{\Lambda} \subset \Sigma$ be the $\sigma$-algebra generated by the spins in $\Lambda$. The "free measure," $\mu_{0}$, is a probability measure on $(\Omega, \Sigma)$ defined as the product of uniform probability measures on $S_{N}$. Let $\mu_{\left.0\right|_{A}} \equiv \mu_{\left.0\right|_{\Sigma_{A}}}$.

Let $\mu$ be a probability measure on $(\Omega, \Sigma)$. For a measurable function $F$, its expectation in the measure $\mu$ is denoted by $\mu(F)$. We set $\mu\left(F, F^{\prime}\right) \equiv \mu\left(F F^{\prime}\right)$ $-\mu(F) \mu\left(F^{\prime}\right)$.

We consider a spin system with a Hamilton function of the following form:

$$
H \equiv-\sum_{\left[\begin{array}{c}
i, j \\
a, b
\end{array}\right]} J_{i j}^{a b} \sigma_{i}^{a} \sigma_{j}^{b}-\sum_{i, a} \underline{h}_{i}^{a} \sigma_{i}^{a} .
$$


The exchange couplings $J_{i j} \equiv\left(J_{i j}^{a b}, a, b=1, \ldots, N\right), i, j \in \mathbb{Z}^{d}$ are defined by

$$
J_{i j}^{a b} \equiv \frac{1}{2} n_{i} G_{i j}^{a b} n_{j}
$$

with $n_{i} \in[0,1]$, and

$$
G_{i j}^{a b} \equiv \frac{1}{(2 \pi)^{d}} \int_{(-\pi, \pi)^{d}} d_{d} q e^{i q(i-j)} \hat{G}^{a b}(q),
$$

where $\hat{G}^{a b}(q)$ is a positive definite $N \times N$ matrix. Furthermore, we assume that (in the sense of quadratic forms)

$$
\widehat{G}^{a b}(q) \leqq C \delta^{a b}
$$

with a constant $0<C<\infty$ independent of $q \in(-\pi, \pi)^{d}$. We denote by $\|\hat{G}\|$ the smallest value of $C$ for which (1.8) holds, i.e.

$$
\|\widehat{G}\| \equiv \min \{C:(1.8) \text { holds }\} .
$$

The external magnetic field $\underline{h}_{i} \equiv\left(\underline{h}_{i}^{a}: a=1, \ldots, N\right), i \in \mathbb{Z}^{d}$ is given by $\underline{h}_{i} \equiv n_{i} h_{i}$, for some $h_{i} \equiv\left(h_{i}^{a}: \in \mathbb{R}, a=1, \ldots, N\right) ; i \in \mathbb{Z}^{d}$.

It is assumed that $\left(n_{i}, i \in \mathbb{Z}^{d}\right)$, and $\left(h_{i}, i \in \mathbb{Z}^{d}\right)$, are independent random variables. A translation invariant probability measure, $E$, on $\mathbb{I} \equiv[0,1]^{\mathbb{Z}^{d}}$ (respectively $\varrho$ on $\left.\mathbb{h} \equiv\left(\mathbb{R}^{N}\right)^{\mathbb{Z}^{d}}\right)$ describes the distribution of the $n$-variables (the one of the external magnetic field variables, $h$, respectively). We restrict our attention to measures $\varrho$ with $\varrho\left(h_{i}^{2}\right)<\infty$.

Note that the class $J_{0}$ of interactions $\left(J_{i j}\right)$ defined by (1.6)-(1.8) contains all the classical short range interactions, i.e. interactions for which

$$
\sum_{j \in \mathbb{Z}^{d}}\left|J_{i j}^{a b}\right|<\infty,
$$

as well as long range interactions which do not satisfy (1.10), but which satisfy

$$
\left|\sum_{j \in \mathbb{Z}^{d}} J_{i j}^{a b}\right|<\infty, \quad E \text {-a.e. . }
$$

In particular, the class $J_{0}$ contains the interactions of RKKY type for which (1.11) is fulfilled, but (1.10) does not hold, $E-$ a.e.. In dimension $d=3$, these interactions are given by

$$
G_{i j}^{a b}:=g^{a b} \frac{1}{|i-j|+\lambda}\left(\frac{k_{F}|i-j| \cos k_{F}|i-j|+\sin k_{F}|i-j|}{k_{F}|i-j|^{3}}\right)
$$

for some constants $0<k_{F}, \lambda<\infty$, and a positive definite matrix $g^{a b}$. (For other examples see Appendix 1).

Note that if an interaction $J \equiv\left(J_{i j}\right)$, given by $G \equiv\left(\hat{G}^{a b}\right)$, is in $J_{0}$ then also the interactions $J^{\prime}$ defined by

$$
\left(\widehat{G}^{\prime}\right)^{a b} \equiv(\|\widehat{G}\|+C) \delta^{a b}-\widehat{G}^{a b}
$$

for any $0<C<\infty$, belong to $J_{0}$.

An interaction $J \in J_{0}$ is called weakly ferromagnetic iff

$$
\sum_{j \in \mathbb{Z}^{a} \mid i} J_{i j}^{a b}>0,
$$

for all $i \in \mathbb{Z}^{d} ; a, b=1, \ldots, N$ (see Appendix 1 for examples). 
The finite-volume pressure $p_{A}, \Lambda \in \mathscr{F}$, is defined by

$$
p_{\Lambda}(\beta, n h):=\frac{1}{|\Lambda|} \ln \mu_{0} e^{-\beta H_{\Lambda}},
$$

with $\beta \in \mathbb{R}^{+}, n \in \mathbb{I}$ and $h \in \mathbb{T}$

Our first result is the following:

Proposition 1. Let $E$ and $\varrho$ be translation invariant probability measures on II, th, respectively, with

$$
\varrho\left(h_{i}^{2}\right)<\infty .
$$

Then, for any interaction $J \in J_{0}$ and any $\beta \geqq 0$, the thermodynamic limit for the pressure in zero magnetic field

$$
p(\beta, n, 0) \equiv \lim _{\mathscr{F}_{0}} p_{\Lambda}(\beta, n, 0)
$$

exists and is independent of $n \in \mathbb{I}, E-$ a.e. The same holds for a nonzero magnetic field $h \equiv\left(h_{i}\right), \varrho-$ a.e., if, in addition, one assumes that

$$
0<\beta\|\hat{G}\|<1 .
$$

The limit $p(\beta, n, h)$ is then also independent of $h, \varrho-$ a.e.

For $\Lambda \in \mathscr{F}$, a finite-volume measure $\mu_{\Lambda}^{\tilde{\sigma}}$ with boundary condition $\tilde{\sigma} \in \Omega$ is defined by

$$
\mu_{\Lambda}^{\tilde{\sigma}}(\cdot):=\lim _{\Lambda^{\prime} \in \mathscr{F}_{0}} \delta_{\tilde{\sigma}}\left(\frac{\mu_{\left.0\right|_{\Lambda}}\left(e^{-\beta H_{\Lambda^{\prime}} \cdot}\right)}{\mu_{\left.0\right|_{\Lambda}}\left(e^{\left.-\beta H_{\Lambda^{\prime}}\right)}\right.}\right),
$$

where, for $\Lambda^{\prime} \in \mathscr{F}_{0}$,

$$
H_{\Lambda^{\prime}} \equiv-\sum_{\substack{a, b=1, \ldots, N \\ i, j \in A^{\prime}}} J_{i j}^{a b} \sigma_{i}^{a} \sigma_{j}^{b}-\sum_{\substack{a=1, \ldots, N \\ i \in \Lambda^{\prime}}} \underline{h}_{i}^{a} \sigma_{i}^{a},
$$

and $\delta_{\tilde{\sigma}}$ is the point measure concentrated at $\tilde{\sigma}$. We note that the set

$$
\widetilde{\Omega} \equiv \bigcap_{\mathscr{F}_{0}}\left\{\tilde{\sigma} \in \Omega: \forall i \in \Lambda\left|\sum_{j \in \Lambda^{c}} J_{i j}^{a b} \cdot \tilde{\sigma}_{j}^{b}\right|<\infty\right\}
$$

is not empty, $E-$ a.e., and therefore the family

$$
\mathscr{E} \equiv \mathscr{E}(\beta, J, h) \equiv\left\{\mu_{\Lambda}^{\tilde{\sigma}}: \Lambda \in \mathscr{F}, \tilde{\sigma} \in \widetilde{\Omega}\right\}
$$

is well defined (and in fact forms a "local specification"). We also consider finite volume measures $\mu_{\Lambda}(\Lambda \in \mathscr{F})$ with adiabatic boundary conditions given by

$$
\mu_{\Lambda}(\cdot):=\frac{\mu_{0}\left(e^{-\beta H_{A}} \cdot\right)}{\mu_{0}\left(e^{-\beta H_{A}}\right)} .
$$

Let $\mu^{\tilde{\sigma}}$ be a limit of $\left\{\mu_{\Lambda}^{\tilde{\sigma}}\right\}$, i.e.

$$
\mu^{\tilde{\sigma}}=\lim _{\mathscr{F}_{0}} \mu_{\Lambda}^{\tilde{\sigma}},
$$

for a countable base $\mathscr{F}_{0}$. By weak compactness of the space of probability measures on $(\Omega, \Sigma)$, the infinite volume measure $\mu^{\tilde{\sigma}}$ is well defined (however, in general, may 
depend on $\mathscr{F}_{0}$ ). Similarly, one can find an infinite volume measure as an accumulation point of the sequence $\left\{\mu_{\Lambda} ; \Lambda \in \mathscr{F}_{0}\right\}$. The set of all infinite volume measures corresponding to a given $\mathscr{E} \equiv \mathscr{E}(\beta, J, h)$ is denoted by $\mathscr{G}(\mathscr{E})$. For $A \in \mathscr{F}$, let $N(A)$ be a multiplicity function which, for any $i \in A$, associates a sequence $\left(N_{i, a} \in \mathbb{Z}^{+}, a=1, \ldots, N\right)$ different from $\bar{O} \equiv\left(N_{l, a} \equiv 0, a=1, \ldots, N\right)$ and, for all $i \in A^{c}$, it is the zero sequence $\bar{O}$.

With a slight abuse of notation we set

$$
\sigma_{A} \equiv \sigma(N(A)) \equiv \prod_{i \in A}\left(\sigma_{i}^{a}\right)^{N_{l, a}},
$$

and, for $j \in \mathbb{Z}^{d}$,

$$
\sigma_{A+j} \equiv \prod_{i \in A}\left(\sigma_{i+j}^{a}\right)^{N_{i, a}} .
$$

For $\mu \in \mathscr{G}(\mathscr{E})$ we define the following generalized susceptibilities:

$$
\chi^{(l)}(A, \mu) \equiv \lim _{\mathscr{F}_{0}} \frac{1}{|\Lambda|} \sum_{i, j \in \Lambda}\left(\mu\left(\sigma_{A+i}, \sigma_{A+j}\right)\right)^{l}
$$

with $l=1,2$.

Proposition 2. Let $J \in J_{0}$ and $h \in \mathrm{lh}$. If

$$
0<\beta\|\hat{G}\|<1
$$

then, for any infinite volume measure $\mu \in \mathscr{G}(\mathscr{E})$ with $\mathscr{E}=\mathscr{E}(\beta, J, h)$, the susceptibilities $\chi^{(l)}(A, \mu)$ are finite.

We define a generalized order parameter by

$$
q(\tilde{\sigma}, A) \equiv \lim _{\tilde{F}_{0}} \frac{1}{|\Lambda|} \sum_{i \in \Lambda}\left(\mu^{\tilde{\sigma}}\left(\sigma_{A+i}\right)\right)^{2} .
$$

In particular, we are interested in the case where $h_{i}^{a}=0\left(i \in \mathbb{Z}^{d}, a=1, \ldots, N\right)$ and the set $A$ is odd, i.e. the volume of the set $\left\{i \in A: N_{i, a}\right.$ odd $\}$ is an odd number. A special case is the Edwards-Anderson order parameter which, for an Ising spin glass, reads

$$
q_{E-A}(\tilde{\sigma}) \equiv \lim _{\mathscr{F}_{0}} \frac{1}{|\Lambda|} \sum_{i \in \Lambda}\left(\mu_{\Lambda}^{\check{\sigma}} \sigma_{i}\right)^{2}
$$

Let $\mathscr{F}_{0}$ be a Fisher sequence.

Proposition 3. Let $J \in J_{0}$ and $h \in \mathbb{l h}$. If

$$
0 \leqq 2 \beta\|\hat{G}\|<1
$$

then, for all functions $\sigma_{A}$ and $l=1,2$, the limits

$$
\left\langle\sigma_{A}\right\rangle_{(l)} \equiv \lim _{\mathscr{F}_{0}} \frac{1}{|\Lambda|} \sum_{i \in A}\left(\mu_{\Lambda} \sigma_{A+i}\right)^{(l)}
$$

exist and are independent of $n \in \mathbb{I}$ and $h \in \mathbb{l}, E \otimes Q-$ a.e.. Moreover,

$$
\lim _{\mathscr{F}_{0}} \frac{1}{|\Lambda|} \sum_{i \in \Lambda}\left(\mu_{\Lambda}^{\grave{\sigma}}\left(\sigma_{A+i}\right)-\mu_{\Lambda}\left(\sigma_{A+i}\right)\right)^{l}=0,
$$


for $\mu$-a.a. $\tilde{\sigma} \in \widetilde{\Omega}$ and any $\mu \in \mathscr{G}(\mathscr{E}(\beta, J, h))$. In particular, if $h=0$ then, for any odd $A$,

$$
q(\tilde{\sigma}, A)=0, \quad \mu \text {-a.e. . }
$$

Propositions 1-3 provide a complete description of spin glass systems in the high temperature region.

The case where the measure $E$ is concentrated on the set $\mathbb{I}_{0} \equiv\{0,1\}^{\mathbb{Z}^{d}}$ is of special interest. Then $E$ describes the density of magnetic atoms (e.g. Fe, Ma) inserted in a host nonmagnetic material (e.g. $\mathrm{Au}, \mathrm{Cu}$ ). The above propositions state that, above some temperature $\beta_{0}^{-1}$ determined by the interaction $J \in J_{0}$, a spin system stays in the disordered phase and its thermodynamic behaviour is independent of the sample, $n$, of magnetic atoms chosen. Note that $\beta_{0}^{-1} \equiv\|\hat{G}\|$ is just the mean field critical temperature, for standard examples of ferromagnetic spin systems.

The proofs of our propositions are essentially the same for any choice of the number, $N$, of spin components. Therefore, to simplify our notation, we shall only consider the Ising models, i.e. $\sigma_{i}= \pm 1$ and $\hat{G}$, defining an interaction $J$, is just a positive, bounded function on $(-\pi, \pi)^{d}$. Without loss of generality, we can and do assume that $0<\varepsilon<\hat{G}(q)$, for some constant $\varepsilon>0$. We also note that $\|\hat{G}\|=\|\hat{G}(\cdot)\|_{\infty}$.

\section{The Thermodynamic Limit of the Pressure}

In this section we prove Proposition 1. Using the assumption that

$$
G_{i j} \equiv \frac{1}{(2 \pi)^{d}} \int d_{d} q e^{i q(i-j)} \widehat{G}(q)
$$

with

$$
\|\hat{G}\|<\infty,
$$

our Hamilton function for a system in a region $\Lambda \in \mathscr{F}$ can be written as follows:

$$
\begin{aligned}
H_{\Lambda} & \equiv-\frac{1}{2} \sum_{i, j \in \Lambda} G_{i j} n_{i} n_{j} \sigma_{i} \sigma_{j}-\sum_{i \in \Lambda} h_{i} n_{i} \sigma_{i} \\
& =-\frac{1}{2} \frac{1}{(2 \pi)^{d}} \int d_{d} q \hat{G}(q)\left|\sum_{j \in A} e^{i q j} n_{j} \sigma_{j}\right|^{2}-\sum_{i \in \Lambda} h_{i} n_{i} \sigma_{i} .
\end{aligned}
$$

This yields the bound

$$
\left|H_{\Lambda}\right| \leqq\left(\frac{1}{2}\|\hat{G}\|+\frac{1}{|\Lambda|} \sum_{i \in \Lambda}\left|h_{i}\right|\right) \cdot|\Lambda|,
$$

which implies

$$
0 \leqq p_{\Lambda}(\beta, J, h) \equiv \frac{1}{|\Lambda|} \ln \mu_{0} e^{-\beta H_{\Lambda}} \leqq \frac{\beta}{2}\|\hat{G}\|+\beta \frac{1}{|\Lambda|} \sum_{i \in \Lambda}\left|h_{i}\right|
$$

(The lower bound follows from symmetry of the product free measure $\mu_{0}$ and Jensen's inequality.) 
By assumption, $\left(h_{i}, i \in \mathbb{Z}^{d}\right)$ are independent random variables with translation invariant distribution $\varrho$ satisfying

$$
\varrho\left(h_{i}^{2}\right)<\infty .
$$

Therefore, by the law of large numbers,

$$
\frac{1}{|\Lambda|} \sum_{i \in \Lambda}\left|h_{i}\right| \underset{\mathscr{F}_{0}}{\longrightarrow} \varrho\left|h_{0}\right|, \quad \varrho-\text { a.e. }
$$

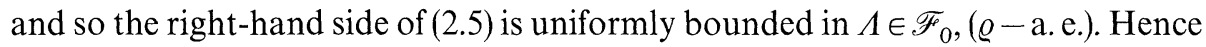
we can always select a convergent subsequence $\left\{p_{\Lambda}(\beta, J, h): \Lambda \in \mathscr{F}_{0}^{\prime}\right\}$.

Now we set $h \equiv 0$ and show that, for any $\mathscr{F}_{\text {exp }}$, the sequence $\left\{p_{A}(\beta, J, 0): \Lambda \in \overline{\mathcal{F}}_{\text {exp }}\right\}$ converges $E$-a.e. to a nonrandom limit. By Jensen's inequality, we have, for any $\Lambda_{k+1} \in \mathscr{F}_{\text {exp }}$,

$$
p_{\Lambda_{k+1}}(\beta, J, 0) \geqq \frac{1}{L^{d}} \sum_{l=1, \ldots, L^{d}} p_{\Lambda_{k}^{(l)}}(\beta, J, 0) .
$$

The definition of an exponential sequence $\mathscr{F}_{\exp }$ implies that $\Lambda_{k}^{(l)} \cap \Lambda_{k}^{\left(l^{\prime \prime}\right)}=\emptyset$, for $l \neq l^{\prime}$, and since by our assumptions $\left\{n_{i}: i \in \mathbb{Z}^{d}\right\}$ are independent and identically distributed, so are $\left\{p_{\Lambda_{k}^{(l)}}: l=1, \ldots, L^{d}\right\}$ independent, identically distributed random variables. This, together with (2.8), shows that the sequence $\left\{E p_{A}(\beta, J, 0): \Lambda \in \mathscr{F}_{\text {exp }}\right\}$ is increasing and our bound (2.5) assures its convergence. (In particular, we obtain convergence of the sequence of finite volume pressures for a translation-invariant interaction $J_{i j} \equiv G_{i j}$ and zero external magnetic field, $h$.)

By iteration of (2.8) and application of the subadditive ergodic theorem, we conclude as in [13] that:

$$
p(\beta, J, 0) \equiv \lim _{\mathscr{F}_{\exp }} E p_{\Lambda}(\beta, J, 0),
$$

exists, and

$$
p(\beta, J, 0)=\lim _{\mathscr{F}_{\exp }} p_{\Lambda}(\beta, J, 0), \quad E \text {-a.e. . }
$$

The simple arguments involving Jensen's inequality allow us to extend (2.9) and (2.10) to more general sequences, $\mathscr{F}_{0}$. This completes the proof of the first claim of Proposition 1.

To include an arbitrary external magnetic field, let us note that, for any $\Lambda \in \mathscr{F}$,

$$
p_{\Lambda}(\beta, J, h)=p_{\Lambda}(\beta, J, 0)+\int_{0}^{1} d t \frac{1}{|\Lambda|} \sum_{i \in \Lambda} h_{i} n_{i} \mu_{\Lambda, t}\left(\sigma_{i}\right),
$$

where the measure $\mu_{A, t}$ is given by (1.22), with magnetic field $\left(t \cdot h_{i}\right)$, instead of $\left(h_{i}\right)$. It follows from the arguments in the proof of (1.30) in Proposition 3 (see Sect. 4) that if

$$
0 \leqq \beta\|\hat{G}\|<1
$$

and $\varrho\left(h_{i}^{2}\right)<\infty$ then, for any $t \in[0,1]$, the sequence

$$
\left\{\frac{1}{|\Lambda|} \sum_{i \in \Lambda} h_{i} n_{i} \mu_{\Lambda, t}\left(\sigma_{i}\right): \Lambda \in \mathscr{F}_{0}\right\}
$$


converges to a nonrandom limit. This together with (2.9) and (2.10) concludes the proof of Proposition 1.

Remarks. a) Note that the positivity of the molecular field for spin systems, i.e.

$$
\mu_{\Lambda}\left(\sigma_{i}\left[\sum_{j \in \mathbb{Z}^{d} \backslash i} J_{i j} \sigma_{j}+h_{i}\right]\right) \geqq 0,
$$

implies the bound

$$
p(\beta, J, h) \leqq\left. p(\beta, J, h)\right|_{\left\{n_{i} \equiv 1\right\}} .
$$

b) We remark that the existence of a nonrandom infinite-volume pressure, $p(\beta, J, 0)$, can also be proven for interactions, $J$, in a class $J_{1}$ defined by

$$
G_{i j} \equiv-\frac{1}{(2 \pi)^{d}} \int \widehat{G}(d q) e^{i q(i-j)}
$$

where $\hat{G}(d q)$ is an arbitrary finite non-negative measure. Then the minus sign in (2.15) assures the trivial bound from above,

$$
p_{\Lambda}(\beta, J, 0) \leqq 1
$$

whereas the symmetry of $\mu_{0, \Lambda}$ together with Jensen's inequality, yields the lower bound

$$
-\beta \frac{1}{(2 \pi)^{d}} \int \hat{G}(d q) \leqq p_{\Lambda}(\beta, J, 0) .
$$

The same arguments, based on Jensen's inequality and the subadditive ergodic theorem, as before, prove our claim. Note that the class $J_{1}$ contains interactions which do not decay at infinity, e.g.

$$
G_{i j}=-\cos \left(q_{0}(i-j)\right) \text {. }
$$

For such an interaction, one can expect that thermodynamics is full of pathologies, therefore we shall only consider interactions from class $J_{0}$.

c) It is possible to extend our results to quantum spin systems.

\section{A Field Picture of Spin Systems}

Let $\phi \equiv\left(\phi_{i} \in \mathbb{R}: i \in \mathbb{Z}^{d}\right)$ be a random field on a probability space $\left(\mu_{G}, \mathbb{R}^{\mathbb{Z}^{d}}, \mathscr{B}\right)$, where $\mu_{G}$ is a Gaussian measure with mean zero and covariance

$$
G_{i j} \equiv \frac{1}{(2 \pi)^{d}} \int_{(-\pi, \pi)^{d}} d_{d} q e^{i q(i-j)} \hat{G}(q)
$$

and $\mathscr{B}$ denotes the Borel $\sigma$-algebra in $\mathbb{R}^{\mathbb{Z}^{d}}$. It is assumed that

$$
0<\varepsilon \leqq \widehat{G}(q) \leqq\|\widehat{G}\|_{\infty}<\infty,
$$

for some constant $\varepsilon>0$. Therefore $\hat{G}(q)^{-1}$ is a well defined (positive and bounded) function, and its Fourier transform, $G^{-1}$, belongs to $l_{2}\left(\mathbb{Z}^{d}\right)$. For any function $f \equiv\left(f_{i} \in \mathbb{R}: i \in \mathbb{Z}^{d}\right) \in l_{2}\left(\mathbb{Z}^{d}\right)$ we define

$$
\phi(f):=\sum_{i \in \mathbb{Z}^{d}} \phi_{i} f_{i}
$$


By our assumptions, $\exp (\phi(f)) \in L_{p}\left(\mu_{G}\right)$ for $1<p<\infty$. In particular this holds for $f(j) \equiv G_{i j}^{-1}$.

The following identity (due to M. Kac [3]) will play an important rôle: For $\Lambda \in \mathscr{F}$,

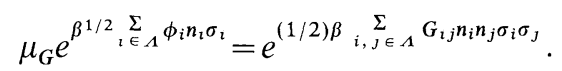

We define a probability measure $\mu_{\Lambda}^{\tilde{\sigma}}$ on $\left(\mathbb{R}^{\mathbb{Z}^{d}}, \mathscr{B}\right) \times\left(\Omega, \Sigma_{\Lambda}\right)$ by

$$
\mu_{\Lambda}^{\tilde{\sigma}}(\cdot) \equiv \frac{1}{Z_{\Lambda}^{\tilde{h}}} \mu_{G} \otimes \mu_{0}\left[e^{\beta^{1 / 2} \sum_{i \in \Lambda}\left(\phi_{i}+\beta^{1 / 2} \tilde{h}_{\imath}\right) n_{\imath} \sigma_{\imath}} \cdot\right],
$$

where $Z_{A}^{\tilde{h}}$ is a normalization factor and

$$
\tilde{h}_{i} \equiv h_{i}+\sum_{j \in \Lambda^{c}} G_{i j} n_{j} \tilde{\sigma}_{j} \equiv h_{i}+h_{i}(\tilde{\sigma}) .
$$

Using (3.4) we see that, for $A \subseteq \Lambda$,

$$
\mu_{\Lambda}^{\tilde{\sigma}}\left(\sigma_{A}\right)=\delta_{\tilde{\sigma}}\left(\frac{\mu_{\left.0\right|_{\Lambda}}\left(e^{-\beta H_{\Lambda}(\sigma)} \sigma_{A}\right)}{\mu_{\left.0\right|_{\Lambda}} e^{-\beta H_{\Lambda}(\sigma)}}\right) .
$$

On the other hand

where

$$
\mu_{\Lambda}^{\tilde{\sigma}}(F(\phi))=\frac{\mu_{G} e^{U_{\Lambda}} F(\phi)}{\mu_{G} e^{U_{\Lambda}}},
$$

$$
U_{\Lambda}(\phi) \equiv \sum_{i \in \Lambda} \ln \operatorname{ch}\left(\beta^{1 / 2}\left(\phi_{i}+\beta^{1 / 2} \tilde{h}_{i}\right) n_{i}\right) .
$$

The measure formally obtained from (3.5) by putting $\left\{n_{j} \equiv 0: j \in \Lambda^{c}\right\}$ is denoted by $\mu_{\Lambda}$. The lemma proven below shows that the expectations (3.8), for $F(\cdot)$ an arbitrary polynomial, uniquely determines the expectations (3.7).

Lemma 3.1. For any $A, B \subseteq \Lambda$

$$
\mu_{\Lambda}^{\tilde{\sigma}}\left(\prod_{j \in A} \phi\left(G_{j}^{-1}\right)\right)=\sum_{x \subseteq A} \mu_{G}\left(\prod_{j \in A \backslash x} \phi\left(G_{j}^{-1}\right)\right) \beta^{\frac{|x|}{2}} n_{x} \mu_{\Lambda}^{\tilde{\sigma}}\left(\sigma_{x}\right),
$$

and

$$
\begin{aligned}
\mu_{A}^{\tilde{\sigma}}\left(\prod_{i \in A} \phi\left(G_{i}^{-1}\right), \prod_{j \in B} \phi\left(G_{j}^{-1}\right)\right)= & \sum_{\substack{x \subseteq A \\
y \subseteq B}}\left(\mu_{G} \prod_{i \in A \backslash x} \phi\left(G_{i}^{-1}\right)\right)\left(\mu_{G} \prod_{j \in B \backslash y} \phi\left(G_{j}^{-1}\right)\right) \\
& \times \beta^{\frac{|x|+|y|}{2}} n_{x} n_{y} \mu_{\Lambda}^{\tilde{\sigma}}\left(\sigma_{x}, \sigma_{y}\right) \\
& +\sum_{\substack{x \subseteq A \\
y \subseteq B}} \mu_{G}\left(\prod_{i \in A \backslash x} \phi\left(G_{i}^{-1}\right), \prod_{j \in B \backslash y} \phi\left(G_{j}^{-1}\right)\right. \\
& \times \beta^{\frac{|x|+|y|}{2}} n_{x} n_{y} \mu_{\Lambda}^{\tilde{\sigma}}\left(\sigma_{x} \sigma_{y}\right) .
\end{aligned}
$$

The proof is a straightforward application of integration by parts in the Gaussian measure $\mu_{G}$. In particular, for $|A|,|B|=1$, we get

$$
\beta^{1 / 2} n_{i} \mu_{\Lambda}^{\tilde{\sigma}}\left(\sigma_{i}\right)=\mu_{\Lambda}^{\tilde{\sigma}} \phi\left(G_{i}^{-1}\right)
$$


and

$$
\beta n_{i} n_{j} \mu_{\Lambda}^{\tilde{\sigma}}\left(\sigma_{i}, \sigma_{j}\right)=\mu_{\Lambda}^{\tilde{\sigma}}\left(\phi\left(G_{i}^{-1}\right), \phi\left(G_{j}^{-1}\right)\right)-G_{i j}^{-1} .
$$

Remark. Note that if $n_{i} \in\{0,1\}$ one can omit $n_{x}$ from the formulas (3.10)-(3.13). Let $m^{2} \geqq 0$ be such that

$$
0 \leqq m^{2}\|\hat{G}\|<1
$$

Define

$$
\hat{G}^{ \pm}:=\left(1 \mp m^{2} \hat{G}\right)^{-1} \hat{G},
$$

and define $G^{ \pm}$to be the Fourier transform of $\widehat{G}^{ \pm}$. Let $\mu_{G^{ \pm}}$be the Gaussian measure on $\left(\mathbb{R}^{\mathbb{Z}^{d}}, \mathscr{B}\right)$ with mean zero and covariance $G^{ \pm}$. For some measurable real functions $\left\{U_{i}(\cdot)\right\}_{i \in \mathbb{Z}^{d}}$ and $\Lambda \in \mathscr{F}$ define

$$
U_{\Lambda}(\phi) \equiv \sum_{i \in \Lambda} U_{i}\left(\phi_{i}\right)
$$

Lemma 3.2 (Brascamp-Lieb inequalities [4]). If the functions

$$
V_{i}^{( \pm)}(y) \equiv \pm \frac{1}{2} m^{2} y^{2}+U_{i}(y)
$$

are convex/concave then, for any $f \in l_{2}\left(\mathbb{Z}^{d}\right)$ and any $k \in \mathbb{N}$,

$$
\mu_{G^{-}}|\phi(f)|^{k} \leqq \frac{\mu_{G} e^{U_{\Lambda}}|\phi(f)|^{k}}{\mu_{G} e^{U_{\Lambda}}} \leqq \mu_{G^{+}}|\phi(f)|^{k} .
$$

Proof. By assumption the function

$$
V_{\Lambda^{\prime}}^{-}(\phi) \equiv-\frac{1}{2} m^{2} \sum_{i \in \Lambda^{\prime}} \phi_{i}^{2}+U_{\Lambda}(\phi)
$$

is concave, for any $\Lambda \in \mathscr{F}, \Lambda^{\prime} \in \mathscr{F}, \Lambda^{\prime} \supseteqq \Lambda$. Introducing a Gaussian measure

$$
\mu_{G^{+}, \Lambda^{\prime}}(\cdot) \equiv \frac{\mu_{G}\left(e^{+\frac{m^{2}}{2} \sum_{i \in A^{\prime}} \phi_{i}^{2}} \cdot\right)}{\mu_{G}\left(e^{+\frac{m^{2}}{2} \sum_{i \in A^{\prime}} \phi_{i}^{2}}\right)},
$$

one can write

$$
\frac{\mu_{G}\left(e^{U_{\Lambda}(\phi)} \cdot\right)}{\mu_{G}\left(e^{U_{\Lambda}(\phi)}\right)}=\frac{\mu_{G^{+}, \Lambda^{\prime}}\left(e^{V_{\bar{\Lambda}^{\prime}}(\phi)} \cdot\right)}{\mu_{G^{+}, \Lambda^{\prime}}\left(e^{V_{\bar{\Lambda}^{\prime}}(\phi)}\right)} .
$$

Since, by our assumptions, $e^{V_{\bar{A}^{\prime}}(\phi)}$ is a log concave function, it follows from the Brascamp-Lieb inequalities [4] that, for any $f \in l_{2}\left(\mathbb{Z}^{d}\right)$ and $k \in \mathbb{N}$,

$$
\frac{\mu_{G} e^{U_{\Lambda}(\phi)}|\phi(f)|^{k}}{\mu_{G} e^{U_{\Lambda}(\phi)}} \leqq \mu_{G^{+}, \Lambda^{\prime}}|\phi(f)|^{k} .
$$

Now, using the fact that $\mu_{G^{+}, \Lambda^{\prime}}$ converges to $\mu_{G^{+}}$, as $\Lambda^{\prime} \uparrow \mathbb{Z}^{d}$, we arrive at the second inequality in (3.18). The proof of the lower bound in (3.18) is similar.

Lemma 3.2 is our main technical tool for what follows. As an immediate consequence it yields the following 
Corollary 3.3. Let $f \in l_{2}\left(\mathbb{Z}^{d}\right)$ and $k \in \mathbb{N}$.

a) If

$$
U_{i}(y)=\ln \operatorname{ch}\left(\beta^{1 / 2}\left(y+\beta^{1 / 2} \tilde{h_{i}}\right) n_{i}\right)
$$

and

$$
0 \leqq \beta\|\widehat{G}\|<1,
$$

then, for

$$
\begin{gathered}
\widehat{G}^{+} \equiv(1-\beta \widehat{G})^{-1} \widehat{G} \\
\mu_{G}|\phi(f)|^{k} \leqq \frac{\mu_{G} e^{U_{\Lambda}}|\phi(f)|^{k}}{\mu_{G} e^{U_{A}}} \leqq \mu_{G^{+}}|\phi(f)|^{k}
\end{gathered}
$$

b) If

$$
U_{i}(y)=\lambda \cos \beta^{1 / 2} y
$$

and

$$
0 \leqq \lambda \beta\|\widehat{G}\|<1
$$

then, for

$$
G^{ \pm} \equiv(1 \mp \lambda \beta \hat{G})^{-1} \hat{G},
$$

the inequalities (3.18) hold.

\section{Cluster Properties of Spin Systems at High Temperature}

We begin with the following general fact which is model-independent.

Proposition 4.1. Suppose that

$$
\sum_{i, j \in \Lambda} f_{i} f_{j} \mu_{\Lambda, 2 \beta, h=0}\left(\sigma_{i}, \sigma_{j}\right) \leqq C \sum_{i} f_{i}^{2}
$$

for some constant $0<C<\infty$ independent of $\Lambda \in \mathscr{F}, \tilde{\sigma} \in \widetilde{\Omega}, n \in \mathbb{I}$ and $f \in l_{2}\left(\mathbb{Z}^{d}\right)$. Then, for any $A \in \mathscr{F}$,

$$
\sum_{\substack{i, j: A+i \subset \Lambda \\ A+j \subset A}} f_{i} f_{j} \mu_{\Lambda, \beta}^{\tilde{\sigma}}\left(\sigma_{A+i}, \sigma_{A+j}\right) \leqq C(A) \sum_{i: A+i \subset A} f_{i}^{2}
$$

for some constant $0<C(A)<\infty$ independent of $\Lambda \in \mathscr{F}, \tilde{\sigma} \in \Omega, \quad n \in \mathbb{I}$ and $f \in l_{2}\left(\mathbb{Z}^{d}\right)$.

Proof. For $\sigma_{l}, \bar{\sigma}_{l} \in\{-1,1\}, l \in \mathbb{Z}^{d}$ define

$$
q_{l}=\frac{1}{2}\left(\sigma_{l}+\bar{\sigma}_{l}\right), \quad p_{l}=\frac{1}{2}\left(\sigma_{l}-\bar{\sigma}_{l}\right) .
$$

We note that

$$
p_{l} \neq 0 \quad \text { iff } \quad q_{l}=0 .
$$

For $A \in \mathscr{F}$, we set $q_{A} \equiv \prod_{i \in A} q_{i}$ and $p_{A} \equiv \prod_{i \in A} p_{i}$. 
Then, for any $f \in l_{2}\left(\mathbb{Z}^{d}\right)$, and $A \in \mathscr{F}$, we have that

$$
\begin{aligned}
& \sum_{\substack{A+i \subset \Lambda \\
A+j \subset A}} f_{i} f_{j} \mu_{\Lambda}^{\tilde{\sigma}} \otimes \bar{\mu}_{\Lambda}^{\tilde{\sigma}}\left(q_{A+i} q_{A+j} p_{i} p_{j}\right) \\
& \quad=\mu_{\Lambda}^{\tilde{\sigma}} \otimes \bar{\mu}_{\Lambda}^{\tilde{\sigma}}\left(\sum_{\substack{A+i \subset \Lambda \\
A+j \subset \Lambda}} f_{i} q_{A+i} f_{j} q_{A+j} M_{\Lambda}\left(p_{i} p_{j} \mid \Sigma_{q}\right)\right),
\end{aligned}
$$

where $\bar{\mu}_{\Lambda}^{\tilde{\sigma}}=\mu_{\Lambda}^{\tilde{\sigma}}$ and $M_{\Lambda}\left(\cdot \mid \Sigma_{q}\right)$ denotes the conditional expectation, associated with the measure $\mu_{\Lambda}^{\tilde{\sigma}} \otimes \bar{\mu}_{\Lambda}^{\tilde{\sigma}}$, with respect to the $\sigma$-algebra, $\Sigma_{q}$, generated by $\left\{q_{i}\right\}$ variables. This conditional expectation is independent of $\tilde{\sigma} \in \widetilde{\Omega}$, and, using (4.4) for any $B \subset A$, one gets:

$$
M_{\Lambda}\left(p_{B} \mid \Sigma_{q}\right)=\frac{\mu_{\left.0\right|_{\Lambda}}\left(e^{-2 \beta H_{\Lambda}(\sigma \mid q)} \sigma_{B}\right)}{\mu_{\left.0\right|_{\Lambda}}\left(e^{-2 \beta H_{\Lambda}(\sigma \mid q)}\right)}
$$

with

$$
H_{\Lambda}(\sigma \mid q) \equiv-\frac{1}{2} \sum_{i, j \in \Lambda} G_{i j} n_{i}(q) n_{j}(q) \sigma_{i} \sigma_{j}
$$

where

$$
n_{i}(q)= \begin{cases}n_{i} & \text { if } q_{i}=0 \\ 0 & \text { otherwise }\end{cases}
$$

We note that the measure on the right-hand side of (4.6) is just $\mu_{\Lambda, 2 \beta, h=0}$, with a given $\left\{n_{i}(q)\right\}$. Therefore, using our assumption (4.1), we have that

$$
\begin{aligned}
& \sum_{\substack{A+i \subset A \\
A+j \subset A}}\left(f_{i} q_{A+i}\right)\left(f_{j} q_{A+j}\right) M_{A}\left(p_{i} p_{j} \mid \Sigma_{q}\right) \\
& \quad \leqq C \sum_{A+i \subset A} f_{i}^{2} q_{A+i}^{2} \leqq C \sum_{A+i \subset A} f_{i}^{2} .
\end{aligned}
$$

The inequality (4.9), together with (4.5), implies that

$$
\sum_{\substack{A+i \subset \Lambda \\ A+j \subset \Lambda}} f_{i} f_{j} \mu_{\Lambda}^{\tilde{\sigma}} \otimes \bar{\mu}_{\Lambda}^{\tilde{\sigma}}\left(q_{A+i} q_{A+j} p_{i} p_{j}\right) \leqq C(A) \sum_{A+i \subset \Lambda} f_{i}^{2} .
$$

Now, (4.2) follows from (4.10) by the same arguments as in [14] (see proof of Theorem 1).

Returning to the old variables, $\sigma_{l}, \bar{\sigma}_{l}$, on the left-hand side of (4.10) and observing that the result can be written as a sum of products of the form

$$
\mu_{\Lambda}^{\tilde{\sigma}}\left(\sigma_{B_{1}+i}, \sigma_{B_{2}+j}\right) \mu_{\Lambda}^{\tilde{\sigma}}\left(\sigma_{B_{3}+i}, \sigma_{B_{4}+j}\right)
$$

or of the form

$$
\mu_{\Lambda}^{\tilde{\sigma}}\left(\sigma_{B_{1}+i}, \sigma_{B_{2}+j}\right) \mu_{\Lambda}^{\tilde{\sigma}}\left(\sigma_{B_{3}+i}\right) \mu_{\Lambda}^{\tilde{\sigma}}\left(\sigma_{B_{4}+j}\right),
$$

for some $B_{1}, \ldots, B_{4} \subset A$, we may use induction in the volume $|A|$, and (4.2) follows by an application of the Schwarz inequality for positive definite forms defined as products of $\mu_{\Lambda}^{\tilde{\sigma}}(\cdot, \cdot)$, see [14].

Remark. In our case the measure $\mu_{\Lambda}^{\tilde{\sigma}}$ is not translation-invariant, so we need a simple modification of the arguments in [14] which are based on uniform bounds on spin expectations; see also Lemma 4.4, below. 
Next, we establish a slight generalization of (4.1).

Lemma 4.2. For any $\Lambda \in \mathscr{F}, \tilde{\sigma} \in \widetilde{\Omega}$ and $n \in \mathbb{I}$, if

$$
0 \leqq \beta\|\widehat{G}\|_{\infty}<1
$$

then, for any $f \in l_{2}\left(\mathbb{Z}^{d}\right)$,

$$
\sum_{i, j} f_{i} f_{j} \mu_{\Lambda}^{\tilde{\sigma}}\left(\phi_{i}, \phi_{j}\right) \leqq \sum_{i, j} f_{i} G_{i j}^{+} f_{j}
$$

As a consequence,

$$
\sum_{i, j \in \Lambda} f_{i} f_{j} n_{i} n_{j} \mu_{\Lambda}^{\tilde{\sigma}}\left(\sigma_{i}, \sigma_{j}\right) \leqq\left(1-\beta\|\widehat{G}\|_{\infty}\right)^{-1} \sum_{i \in \Lambda} f_{i}^{2} .
$$

Proof. We have

where

$$
\begin{aligned}
\sum_{i, j} f_{i} f_{j} \mu_{\Lambda}^{\tilde{\sigma}}\left(\phi_{i}, \phi_{j}\right) & =\sum_{i, j} f_{i} f_{j} \mu_{\Lambda}^{\tilde{\sigma}} \otimes \mu_{\Lambda}^{\tilde{\sigma}}\left(\eta_{i} \eta_{j}\right) \\
& =\mu_{\Lambda}^{\tilde{\sigma}} \otimes \bar{\mu}_{\Lambda}^{\tilde{\sigma}} \sum_{i, j} f_{i} f_{j} M_{\Lambda}^{\tilde{\sigma}}\left(\eta_{i} \eta_{j} \mid \Sigma_{\xi}\right),
\end{aligned}
$$

$$
\left.\begin{array}{rl}
\xi_{i} & \equiv \frac{1}{\sqrt{2}}\left(\phi_{i}+\bar{\phi}_{i}\right) \\
\eta_{i} & \equiv \frac{1}{\sqrt{2}}\left(\phi_{i}-\bar{\phi}_{i}\right)
\end{array}\right\}
$$

and $M_{\Lambda}^{\tilde{\sigma}}\left(\cdot \mid \Sigma_{\xi}\right)$ is the conditional expectation, associated with the (field) measure $\mu_{\Lambda}^{\tilde{\sigma}} \otimes \bar{\mu}_{\Lambda}^{\tilde{\sigma}}$, with respect to the $\sigma$-algebra $\Sigma_{\xi}$ generated by the $\left\{\xi_{i}\right\}$ variables.

Since $\mu_{\Lambda}^{\tilde{\sigma}} \equiv \bar{\mu}^{\tilde{\sigma}}$ is given by (3.8) and (3.9),

$$
M_{\Lambda}^{\tilde{\sigma}}\left(\cdot \mid \sum_{\xi}\right)=\frac{\mu_{G}\left(e^{U_{\Lambda}\left(\frac{1}{\sqrt{2}}(\xi+\cdot)\right)+U_{\Lambda}\left(\frac{1}{\sqrt{2}}(\xi-\cdot)\right)}\right)}{\mu_{G}\left(e^{U_{\Lambda}\left(\frac{1}{\sqrt{2}}(\xi+\cdot)\right)+U_{\Lambda}\left(\frac{1}{\sqrt{2}}(\xi-\cdot)\right)}\right)}
$$

Now we observe that the functions

$$
\begin{aligned}
V_{i}^{-}(y) \equiv & -\frac{1}{2} \beta\left(\frac{1}{\sqrt{2}}\left(\xi_{i}+y\right)\right)^{2} \\
& +\operatorname{lnch}\left[\beta^{1 / 2}\left(\left(\frac{1}{\sqrt{2}}\left(\xi_{i}+\mathrm{y}\right)\right)+\beta^{1 / 2} \tilde{h}_{i}\right) n_{i}\right] \\
& -\frac{1}{2} \beta\left(\frac{1}{\sqrt{2}}\left(\xi_{i}-y\right)\right)^{2} \\
& +\ln \operatorname{ch}\left[\beta^{1 / 2}\left(\left(\frac{1}{\sqrt{2}}\left(\xi_{i}-\mathrm{y}\right)\right)+\beta^{1 / 2} \tilde{h}_{i}\right) n_{i}\right] \\
= & -\frac{1}{2} \beta y^{2}+\ln \operatorname{ch}\left[\beta^{1 / 2}\left(\frac{1}{\sqrt{2}}\left(\xi_{i}+\mathrm{y}\right)+\beta^{1 / 2} \tilde{h}_{i}\right) n_{i}\right] \\
& +\ln \operatorname{ch}\left[\beta^{1 / 2}\left(\frac{1}{\sqrt{2}}\left(\xi_{i}-y\right)+\beta^{1 / 2} \tilde{h}_{i}\right) n_{i}\right]-\frac{1}{2} \beta \xi_{i}^{2}
\end{aligned}
$$


are concave. Therefore, for $0 \leqq \beta$ satisfying (4.11), an application of Lemma 3.2 gives

$$
0 \leqq \sum_{i, j} f_{i} f_{j} M_{\Lambda}^{\check{\sigma}}\left(\eta_{i} \eta_{j} \mid \Sigma_{\xi}\right) \leqq \sum_{i, j} f_{i} G_{i j}^{+} f_{j} .
$$

From (4.12) we get, using (3.13) and definition (3.15) of $G^{+}$with $m^{2} \equiv \beta$,

$$
\begin{aligned}
\sum_{i, j \in \Lambda} & f_{i} f_{j} \beta n_{i} n_{j} \mu_{\Lambda}^{\tilde{\sigma}}\left(\sigma_{i}, \sigma_{j}\right) \\
& \leqq \sum_{i, j \in \Lambda} f_{i} f_{j}\left[\left(G^{-1} G^{+} G^{-1}\right)_{i j}-G_{i j}^{-1}\right] \\
& =\beta \sum_{k=0}^{\infty} \beta^{k} \sum_{i j \in \Lambda} f_{i} f_{j} G_{i j}^{k} \\
& \leqq \beta\left(1-\beta\|\hat{G}\|_{\infty}\right)^{-1} \sum_{i \in \Lambda} f_{i}^{2} .
\end{aligned}
$$

This ends the proof of (4.13) and hence of the lemma.

Remark. Considering the square terms in (4.17), multiplied by $n_{i}$ one can see that, in fact (4.13) remains true without factors $n_{i} n_{j}$ on its right-hand side for general $n_{i} \in[0,1]$. (This is of course true if $n_{i} \in\{0,1\}$.)

For $A \in \mathscr{F}$ and a multiplicity function $N(A)$ we define

$$
\phi_{A} \equiv \prod_{i \in A} \phi_{i}^{N_{i}(A)} .
$$

Using Lemma 4.2 and ideas of [14], one can prove the following analogue of Proposition 4.1 for the "field" variables, $\phi$.

Proposition 4.3. For any $\Lambda \in \mathscr{F}, \tilde{\sigma} \in \widetilde{\Omega}$ and $n \in \mathbb{I}$, if

$$
0 \leqq \beta\|\widehat{G}\|_{\infty}<1,
$$

then, for any $A \in \mathscr{F}$ and $f \in l_{2}\left(\mathbb{Z}^{d}\right)$,

$$
\sum_{i, j} f_{i} f_{j} \mu_{\Lambda}^{\tilde{\sigma}}\left(\phi_{A+i}, \phi_{A+j}\right) \leqq C(A) \sum_{i} f_{i}^{2},
$$

where $C(A)$ is a positive, finite constant only depending on $\beta\|\widehat{G}\|_{\infty}$ and the norm of the multiplicity function $|N(A)| \equiv \sum_{i \in \mathbb{Z}^{d}} N_{i}(A)$.

Remark. The same result holds for any measure $\tilde{\mu}_{A}$ defined as a perturbation of the Gaussian measure $\mu_{G}$ considered in Lemma 3.2 and Corollary 3.3, and it holds for any infinite volume measure $\mu \equiv \lim _{\mathscr{F}_{0}} \tilde{\mu}_{\Lambda}$, where $\mathscr{F}_{0}$ is a countable base.

Since, contrary to [14], we deal with nontranslation-invariant measures, we have to use, in the proofs of Propositions 4.1 and 4.3, the following lemma which is of independent interest.

Lemma 4.4. If, for $A \in \mathscr{F}$,

$$
\sum_{\substack{i, j i \\ A+i \subset \Lambda \\ A+j \subset \Lambda}} f_{i} f_{j} \mu_{\Lambda}^{\tilde{\sigma}}\left(\sigma_{A+i}, \sigma_{A+j}\right) \leqq C_{1} \cdot \sum_{i: A+i \subset \Lambda} f_{i}^{2}
$$


for some constant $0<C_{1}<\infty$ independent of $\Lambda \in \mathscr{F}, \tilde{\sigma} \in \widetilde{\Omega}, n \in \mathbb{I}$ and $f \in l_{2}\left(\mathbb{Z}^{d}\right)$, then, for any $B \in \mathscr{F}$,

$$
\sum_{i, j} f_{i} f_{j} \mu_{\Lambda}^{\tilde{\sigma}}\left(\sigma_{A+i}, \sigma_{A+j}\right) \mu_{\Lambda}^{\tilde{\sigma}}\left(\sigma_{B+i}, \sigma_{B+j}\right) \leqq C_{2} \sum_{i} f_{i}^{2}
$$

and

$$
\sum_{i, j} f_{i} f_{j} \mu_{\Lambda}^{\tilde{\sigma}}\left(\sigma_{A+i}, \sigma_{A+j}\right) \mu_{\Lambda}^{\tilde{\sigma}} \sigma_{B+i} \mu_{\Lambda}^{\tilde{\sigma}} \sigma_{B+j} \leqq C_{3} \sum_{i} f_{i}^{2}
$$

Here $C_{2}$ and $C_{3}$ are constants, with $0<C_{2}, C_{3}<\infty$, independent of $\Lambda \in \mathscr{F}$, $\tilde{\sigma} \in \Omega, n \in \mathbb{I}$ and $f \in l_{2}\left(\mathbb{Z}^{d}\right)$, and the summation in (4.24) and (4.25) is restricted by the requirement that $A+i, A+j, B+i, B+j \subset \Lambda$. Moreover, the same results hold for "fields" $\phi$ if, in addition, for any $\ell \in \mathbb{N}$

$$
\mu_{\Lambda}^{\tilde{\sigma}} \phi_{i}^{2 l}<C_{l},
$$

for a constant $0<C_{l}<\infty$ independent of $i \in \mathbb{Z}^{d}$ and all other parameters. (In this case we don't need to restrict the summation over $i, j$.)

Remark. Under the assumptions of Lemma 3.2, the condition (4.25a) is fullfilled. Proof. Since

$$
\begin{aligned}
& \sum_{i, j} f_{i} f_{j} \mu_{\Lambda}^{\tilde{\sigma}}\left(\sigma_{A+i}, \sigma_{A+j}\right) \mu_{\Lambda}^{\tilde{\sigma}}\left(\sigma_{B+i}, \sigma_{B+j}\right) \\
&=\frac{1}{2} \mu_{\Lambda}^{\tilde{\sigma}} \otimes \bar{\mu}_{\Lambda}^{\tilde{\sigma}}\left(\sum_{i, j} f_{i}\left(\sigma_{B+i}-\bar{\sigma}_{B+i}\right) f_{j}\left(\sigma_{B+j}-\bar{\sigma}_{B+j}\right)\right. \\
&\left.\quad \times \mu_{\Lambda}^{\tilde{\sigma}}\left(\sigma_{A+i}, \sigma_{A+j}\right)\right) \\
& \leqq C_{1} \frac{1}{2} \sup _{i} \mu_{\Lambda}^{\tilde{\sigma}} \otimes \bar{\mu}_{\Lambda}^{\tilde{\sigma}}\left(\sigma_{B+i}-\bar{\sigma}_{B+i}\right)^{2} \sum_{i} f_{i}^{2}
\end{aligned}
$$

and

$$
\begin{gathered}
C_{1} \frac{1}{2} \sup _{i} \mu_{\Lambda}^{\tilde{\sigma}} \otimes \bar{\mu}_{\Lambda}^{\tilde{\sigma}}\left(\sigma_{B+i}-\bar{\sigma}_{B+i}\right)^{2} \\
=C_{1} \sup _{i} 2 \mu_{\Lambda}^{\tilde{\sigma}}\left(\sigma_{B+i}^{2}\right)<C_{2}
\end{gathered}
$$

with $0<C_{2}<\infty$ independent of $\Lambda, \tilde{\sigma}, n$ and of $f,(4.24)$ follows. The proof of (4.25) is trivial. The proof in the $\phi$-variables is similar.

Applications of Lemma 4.2, Lemma 4.4 and Proposition 4.1 yield the following bounds on generalized susceptibilities.

Proposition 4.5. If $0 \leqq \beta\|\widehat{G}\|<1$ then, for any $\bar{\Lambda} \leqq \Lambda, \Lambda \in \mathscr{F}, \tilde{\sigma} \in \widetilde{\Omega}$ and $n \in \mathbb{I}$,

$$
0 \leqq \frac{1}{|\bar{\Lambda}|} \sum_{i, j \in \bar{\Lambda}} \mu_{\Lambda}^{\tilde{\sigma}}\left(\sigma_{i}, \sigma_{j}\right) \leqq(1-\beta\|\widehat{G}\|)^{-1}
$$

and

$$
0 \leqq \frac{1}{|\bar{\Lambda}|} \sum_{i, j \in \bar{\Lambda}}\left(\mu_{\Lambda}^{\tilde{\sigma}}\left(\sigma_{i}, \sigma_{j}\right)\right)^{2} \leqq 2(1-\beta\|\hat{G}\|)^{-1}
$$


Moreover, if $0 \leqq 2 \beta\|\hat{G}\|<1$, then for $l=1,2$,

$$
0 \leqq \frac{1}{|\bar{\Lambda}|} \sum_{\substack{i, j \\ A+i, A+j \subset \bar{\Lambda}}}\left(\mu_{\Lambda}^{\tilde{\sigma}}\left(\sigma_{A+i}, \sigma_{A+j}\right)\right)^{l} \leqq C_{l}(A)
$$

for some constant $0<C_{l}(A)<\infty$ independent of $\bar{\Lambda} \subset \Lambda, \Lambda \in \mathscr{F}, \tilde{\sigma} \in \widetilde{\Omega}$ and $n \in \mathbb{I}$. The same results hold for

$$
\mu \equiv \lim _{\mathscr{F}_{0}} \mu_{\Lambda}^{\tilde{\sigma}},
$$

where $\mathscr{F}_{0}$ is a countable base, $\tilde{\sigma} \in \tilde{\Omega}$ or $\tilde{\sigma}=0$.

\section{The Thermodynamic Limit for Order Parameters}

We begin with showing that the thermodynamic limit of correlations of physical observables is independent of boundary conditions in the high temperature region. The assumptions on the interaction $J$ are the same as in Sects. 3 and 4. For $A \in \mathscr{F}$, $\tilde{\sigma} \in \widetilde{\Omega}, l=1,2$, we define

$$
\left\langle\sigma_{A}\right\rangle_{l, \tilde{\sigma}} \equiv \lim _{\mathscr{F}_{0}} \frac{1}{|\Lambda|} \sum_{i: A+i \subset A}\left(\mu_{\Lambda}^{\tilde{\sigma}}\left(\sigma_{A+i}\right)\right)^{l}
$$

and

$$
\left\langle\sigma_{A}\right\rangle_{l} \equiv \lim _{\mathscr{F}_{0}} \frac{1}{|\Lambda|} \sum_{i: A+i \subset \Lambda}\left(\mu_{\Lambda}\left(\sigma_{A+i}\right)\right)^{l}
$$

Proposition 5.1. Let $\mathscr{F}_{0}$ be a Fisher countable base. Let

$$
0 \leqq \beta\|\widehat{G}\|_{\infty}<1,
$$

for $|A|=1$, and

$$
0 \leqq 2 \beta\|\hat{G}\|_{\infty}<1
$$

for $|A| \geqq 2$, then

$$
\left\langle\sigma_{A}\right\rangle_{l, \tilde{\sigma}}=\left\langle\sigma_{A}\right\rangle_{l}, \quad \mu \text {-a.e. }
$$

and

$$
\lim _{\mathscr{F}_{0}} \frac{1}{|\Lambda|} \sum_{i: A+i \subset \Lambda}\left(\mu_{\Lambda}^{\tilde{\sigma}}\left(\sigma_{A+i}\right)-\mu_{\Lambda}\left(\sigma_{A+i}\right)\right)^{l}=0, \quad \mu \text {-a.e. },
$$

for any $\mu \in \mathscr{G}(\mathscr{E})$.

Proof. For $\Lambda \in \mathscr{F}, \tilde{\sigma} \in \widetilde{\Omega}$ and $t \in[0,1]$, we define a measure $\mu_{\Lambda}^{t \tilde{\sigma}}(\cdot)$ as in (3.5), but, instead of $\tilde{h}_{i}$, the magnetic field is given by

$$
\tilde{h}_{i}(t) \equiv h_{i}+t h_{i}(\tilde{\sigma}),
$$

where

$$
h_{i}(\tilde{\sigma}) \equiv \sum_{j \in \Lambda^{c}} G_{i j} n_{j} \tilde{\sigma}_{j}
$$


Then, for

$$
f_{\Lambda}^{(1)}(t) \equiv \frac{1}{|\Lambda|} \sum_{i: A+i \subset \Lambda}\left(\mu_{\Lambda}^{t \tilde{\sigma}}\left(\sigma_{A+i}\right)-\mu_{\Lambda}\left(\sigma_{A+i}\right)\right)
$$

we have

$$
\frac{d}{d t} f_{\Lambda}^{(1)}(t)=\frac{1}{|\Lambda|} \sum_{i: A+i \subset \Lambda} \sum_{j \in \Lambda} \mu_{\Lambda}^{t \tilde{\sigma}}\left(\sigma_{A+i}, \sigma_{j}\right) n_{j} h_{j}(\tilde{\sigma})
$$

An application of the Schwarz inequality for the positive definite form $\mu_{\Lambda}^{t \tilde{\sigma}}(\cdot, \cdot)$ yields the bound

$$
\begin{aligned}
\left|\frac{d}{d t} f_{\Lambda}^{(1)}(t)\right| \leqq & \left(\frac{1}{|\Lambda|} \sum_{\substack{i, j \\
A+i, A+j \subset \Lambda}} \mu_{\Lambda}^{t \tilde{\sigma}}\left(\sigma_{A+i}, \sigma_{A+j}\right)\right)^{1 / 2} \\
& \times\left(\frac{1}{|\Lambda|} \sum_{i, j \in \Lambda} h_{i}(\tilde{\sigma}) h_{j}(\tilde{\sigma}) n_{i} n_{j} \mu_{\Lambda}^{t \tilde{\sigma}}\left(\sigma_{i}, \sigma_{j}\right)\right)^{1 / 2} .
\end{aligned}
$$

Since Proposition 4.5 and Lemma 4.2 also hold for the measures $\mu_{\Lambda}^{t \tilde{\sigma}},(5.10)$ can be bounded by

$$
\left|\frac{d}{d t} f_{\Lambda}^{(1)}(t)\right| \leqq C(A)\left(\frac{1}{|\Lambda|} \sum_{i \in \Lambda} h_{i}(\tilde{\sigma})^{2}\right)^{1 / 2}
$$

for some constant $0<C(A)<\infty$ independent of $\Lambda \in \mathscr{F}, \tilde{\sigma} \in \widetilde{\Omega}, n \in \mathbb{I}$ and $t \in[0,1]$. This implies

$$
\begin{aligned}
& \left|\frac{1}{|\Lambda|} \sum_{i: A+i \subset \Lambda} \mu_{\Lambda}^{\tilde{\sigma}}\left(\sigma_{A+i}\right)-\frac{1}{|\Lambda|} \sum_{i: A+i \subset \Lambda} \mu_{\Lambda}\left(\sigma_{A}\right)\right| \\
& \quad \leqq C(A)\left(\frac{1}{|\Lambda|} \sum_{i \in \Lambda} h_{i}(\tilde{\sigma})^{2}\right)^{1 / 2} .
\end{aligned}
$$

Consider now

$$
f_{\Lambda}^{(2)}(t) \equiv \frac{1}{|\Lambda|} \sum_{i: A+i \subset A}\left(\left(\mu_{\Lambda}^{t \tilde{\sigma}}\left(\sigma_{A+i}\right)\right)^{2}-\left(\mu_{\Lambda}\left(\sigma_{A+i}\right)\right)^{2}\right) .
$$
Using Hölder's inequality, with respect to $\frac{1}{|\Lambda|} \sum_{i \in \Lambda}(\cdot)$ and the fact that $\left|\mu_{\Lambda}^{t \tilde{\sigma}} \sigma_{A+i}\right| \leqq 1$,
we get

$$
\left|f_{\Lambda}^{(2)}(t)\right| \leqq 2^{1 / 2}\left(\frac{1}{|\Lambda|} \sum_{i: A+i \subset \Lambda}\left(\mu_{\Lambda}^{t \tilde{\sigma}}\left(\sigma_{A+i}\right)-\mu_{\Lambda}\left(\sigma_{A+i}\right)\right)^{2}\right)^{1 / 2} \equiv 2^{1 / 2}\left(g_{\Lambda}(t)\right)^{1 / 2} .
$$

For the function $g_{\Lambda}(t)$ we have

$$
\begin{aligned}
\frac{d}{d t} g_{\Lambda}(t)= & 2 \frac{1}{|\Lambda|} \sum_{i: A+i \subset \Lambda} \sum_{j \in \Lambda} \\
& \times\left(\mu_{\Lambda}^{t \tilde{\sigma}}\left(\sigma_{A+i}\right)-\mu_{\Lambda}\left(\sigma_{A+i}\right)\right) \mu_{\Lambda}^{t \tilde{\sigma}}\left(\sigma_{A+i}, \sigma_{j}\right) n_{j} h_{j}(\tilde{\sigma}) .
\end{aligned}
$$

Hence, applying the Schwarz inequality, Proposition 4.5 and Lemma 4.2 for the measure $\mu_{\Lambda}^{t \widetilde{\sigma}}(\cdot)$, we conclude that

$$
\left|\frac{d}{d t} g_{\Lambda}(t)\right| \leqq C\left(g_{\Lambda}(t)\right)^{1 / 2}\left(\frac{1}{|\Lambda|} \sum_{i \in \Lambda} h_{i}(\tilde{\sigma})^{2}\right)^{1 / 2}
$$


for some constant $0<C<\infty$ independent of $\Lambda \in \mathscr{F}, \tilde{\sigma} \in \widetilde{\Omega}, n \in \mathbb{I}$ and $t \in[0,1]$. This yields the following integral inequality:

$$
0 \leqq g_{\Lambda}(t) \leqq C\left(\frac{1}{|\Lambda|} \sum_{i \in \Lambda} h_{i}(\tilde{\sigma})^{2}\right)^{1 / 2}\left(\int_{0}^{t} d t^{\prime} g_{\Lambda}\left(t^{\prime}\right)\right)^{1 / 2} .
$$

Applying the same inequality to $g_{A}\left(t^{\prime}\right)$, and iterating the bound, we find that

$$
\frac{1}{|\Lambda|} \sum_{i: A+i \subset \Lambda}\left(\mu_{\Lambda}^{\tilde{\sigma}} \sigma_{A+i}-\mu_{\Lambda} \sigma_{A+i}\right)^{2} \leqq C^{2} \frac{1}{|\Lambda|} \sum_{i \in \Lambda} h_{i}(\tilde{\sigma})^{2} .
$$

Now the proof of our proposition follows from (5.12), (5.14), and (5.18) and Lemma 5.2 proven below.

Lemma 5.2. If $0 \leqq \beta\|\widehat{G}\|<1$, then, for any measure $\mu \in \mathscr{G}(\mathscr{E})$,

$$
\lim _{\mathscr{F}_{0}} \frac{1}{|\Lambda|} \sum_{i \in \Lambda} h_{i}(\tilde{\sigma})^{2}=0
$$

for some Fisher countable base $\mathscr{F}_{0}$.

Proof. It is sufficient to show that, for some Fisher sequence $\mathscr{F}_{0}$,

$$
\lim _{\mathscr{F}_{0}} \mu \frac{1}{|\Lambda|} \sum_{i \in \Lambda} h_{i}(\sigma)^{2}=0
$$

where

$$
h_{i}(\sigma) \equiv \lim _{\Lambda^{\prime} \in \mathscr{F}_{0}} \sum_{j \in A^{c} \cap \Lambda^{\prime}} G_{i j} n_{j} \sigma_{j} .
$$

By definition of $\mu \in \mathscr{G}(\mathscr{E})$, we have

$$
\mu \equiv \lim _{\mathscr{F}_{0}} \mu_{\Lambda}^{\tilde{\sigma}},
$$

for some $\tilde{\sigma} \in \widetilde{\Omega}$. Therefore application of Lemma 3.1 [see also (3.13)] yields

$$
\begin{aligned}
\beta \mu h_{i}(\sigma)^{2} & =\sum_{j, j^{\prime} \in A^{c}} G_{i j} G_{i j^{\prime}} \beta n_{j} n_{j^{\prime}} \mu\left(\sigma_{j} \sigma_{j^{\prime}}\right) \\
& =\sum_{j, j^{\prime} \in A^{c}} G_{i j} G_{i j^{\prime}}\left(\mu \phi\left(G_{j}^{-1}\right) \phi\left(G_{j^{\prime} .}^{-1}\right)-G_{j j^{\prime}}^{-1}\right) \\
& =\mu\left|\sum_{j} G_{i j} \phi\left(G_{j^{\prime}}^{-1}\right)\right|^{2}-\sum_{j, j^{\prime} \in \Lambda^{c}} G_{i j} G_{j j^{\prime}}^{-1} G_{j^{\prime}} .
\end{aligned}
$$

From this identity and the Brascamp-Lieb inequalities for the measures $\mu$ (i.e. Lemma 3.2, supplemented by some limiting arguments), we get

$$
\begin{aligned}
\mu h_{i}(\sigma)^{2} & \leqq \beta^{-1} \sum_{j, j^{\prime} \in \Lambda^{c}} G_{i j} G_{i j^{\prime}}\left(G_{j}^{-1} G G_{j^{\prime}}^{-1}-G_{j j^{\prime}}^{-1}\right) \\
& \leqq \sum_{n=0}^{\infty} \beta^{n}\left(\sum_{j, j^{\prime} \in A^{c}} G_{i j} G_{i j^{\prime}}\left(G^{n}\right)_{j j^{\prime}}\right) \\
& \leqq(1-\beta\|\hat{G}\|)^{-1} \sum_{j \in A^{c}} G_{i j}^{2} .
\end{aligned}
$$


Therefore the proof of (5.20), and hence the proof of our lemma, follow from the fact that

$$
\lim _{\mathscr{F}_{0}} \frac{1}{|\Lambda|} \sum_{i \in \Lambda} \sum_{j \in \Lambda^{c}} G_{i j}^{2}=0
$$

for a Fisher countable base $\mathscr{F}_{0}$.

From Proposition 5.1, we derive the following corollary.

Corollary 5.3. Let $0 \leqq \beta\|\widehat{G}\|<1$ and

$$
\mu \equiv \lim _{\mathscr{F}_{0}} \mu_{\Lambda}^{\tilde{\sigma}}
$$

for some $\tilde{\sigma} \in \widetilde{\Omega},\left\{h_{i} \equiv 0, i \in \mathbb{Z}^{d}\right\}$ and a countable base $\mathscr{F}_{0}$. Then

$$
q_{E-A} \equiv \lim _{\mathscr{F}_{0}} \frac{1}{|\Lambda|} \sum_{i \in \Lambda}\left(\mu \sigma_{i}\right)^{2}=0 .
$$

Proof. One can see that the measures

$$
\mu_{\Lambda}^{\tilde{\sigma}} \equiv \lim _{\Lambda^{\prime} \in \mathscr{F}_{0}} \mu_{\Lambda}^{\left.\tilde{\sigma}\right|_{\Lambda^{\prime}}}
$$

define the conditional expectations associated with $\mu$, for $\mu$-a.a. $\tilde{\sigma} \in \widetilde{\Omega}$. Using this fact we conclude that

$$
\begin{aligned}
\frac{1}{|\Lambda|} \sum_{i \in \Lambda}\left(\mu \sigma_{i}\right)^{2} & =\frac{1}{|\Lambda|} \sum_{i \in \Lambda}\left(\mu\left(\mu_{\Lambda}^{\tilde{\sigma}} \sigma_{i}\right)\right)^{2} \\
& \leqq \mu \frac{1}{|\Lambda|} \sum_{i \in \Lambda}\left(\mu_{\Lambda}^{\tilde{\sigma}} \sigma_{i}\right)^{2}
\end{aligned}
$$

and an application of Proposition 5.1 completes the proof.

Using the ideas in the proof of Proposition 5.1 we now show the existence of the thermodynamic limit for the physical quantities (5.1) and (5.2). This will complete the proof of Proposition 3 in Sect. 1.

Proposition 5.4. Let $0 \leqq \beta\|\widehat{G}\|<1$, for $|A|=1$, and $0 \leqq 2 \beta\|\widehat{G}\|<1$, for $|A| \geqq 2$. Then, for $l=1,2$ and any Fisher sequence $\mathscr{F}_{0}$,

$$
\left\langle\sigma_{A}\right\rangle_{(l)} \equiv \lim _{\mathscr{F}_{0}} \frac{1}{|\Lambda|} \sum_{i: A+i \subset \Lambda}\left(\mu_{\Lambda} \sigma_{A+i}\right)^{(l)}
$$

exists and is independent of $n \in \mathbb{I}$ and $h \in \mathbb{h}, E \otimes \varrho$-a.e.

Proof. Let $\mathscr{F}_{\exp }$ be a Fisher exponential base of $\mathscr{F}$. Then for $A \in \mathscr{F}$ and $\Lambda\left(N+N_{0}\right) \in \mathscr{\mathscr { F }}_{\text {exp }}, N, N_{0} \in \mathbb{N}$, we have that

$$
\begin{aligned}
= & \left\{\frac{1}{L^{d N}} \sum_{k=1, \ldots, L^{d N}} \frac{1}{\mid \Lambda_{N_{0}}} \sum_{i: A+i \subset \Lambda_{N_{0}}^{(k)}}\right. \\
& \times\left[\left(\mu_{\Lambda\left(N+N_{0}\right)}\left(\sigma_{A+i}\right)\right)^{l}-\left(\mu_{\Lambda_{N_{0}}^{(k)}}\left(\sigma_{A+i}\right)\right)^{l}\right] \\
& +\frac{1}{L^{d N}} \sum_{k=1, \ldots, L^{d N}} \frac{1}{\left|\Lambda_{N_{0}}\right|} \sum_{i: A+i \cap \hat{\partial} \Lambda_{N 0}^{(k)} \neq \emptyset} \\
& \left.\times\left(\mu_{\Lambda\left(N+N_{0}\right)}\left(\sigma_{A+i}\right)\right)^{l}\right\}+\frac{1}{L^{d N}} \sum_{k=1, \ldots, L^{d N}} \frac{1}{\left|\Lambda_{N_{0}}\right|} \sum_{i: A+i \subset \Lambda_{N_{0}}^{(k)}}\left(\mu_{\left.\Lambda_{N_{0}}^{(k)}\left(\sigma_{A+i}\right)\right)^{l} .}\right.
\end{aligned}
$$


We argue that the right-hand side of (5.28) consists of a "small" term, in the curly bracket, and of the sum of uniformly bounded, identically distributed random variables

$$
\frac{1}{\left|\Lambda_{N_{0}}\right|} \sum_{i: A+i \subset \Lambda_{N_{0}}^{(k)}}\left(\mu_{A_{N_{0}}^{(k)}}\left(\sigma_{A+i}\right)\right)^{l} .
$$

(Note that, by definition of $\mathscr{F}_{\text {exp }}$, we have that $\Lambda_{N_{0}}^{(k)} \cap \Lambda_{N_{0}}^{\left(k^{\prime}\right)}=\emptyset$, for $k \neq k^{\prime}$, and by our assumptions, the measures $E$ and $\varrho$ are translation-invariant product probability measures.)

First, we observe that

$$
\frac{1}{L^{d N}} \sum_{k=1, \ldots, L^{a N}} \frac{1}{\left|\Lambda_{N_{0}}\right|} \sum_{i: A+i \cap \hat{\partial} \Lambda_{N_{0}}^{(k)} \neq \emptyset}\left(\mu_{A\left(N+N_{0}\right)}\left(\sigma_{A+i}\right)\right)^{l} \leqq C^{\prime} \frac{\left|\partial \Lambda_{N_{0}}\right|}{\left|\Lambda_{N_{0}}\right|} \leqq e^{-C N_{0}},
$$

for constants $0<C^{\prime}, C<\infty$ independent of $N_{0} \in \mathbb{N}$, (and of $n \in \mathbb{I}$ and $h \in \mathbb{h}$ ).

Moreover, for $l=1,2$, we use the Schwarz and the Hölder inequalities, to conclude that

$$
\begin{aligned}
& \left|\frac{1}{\left|\Lambda_{N_{0}}\right|} \sum_{i: A+i \subset \Lambda_{N_{0}}^{(k)}}\left(\left(\mu_{A\left(N+N_{0}\right)}\left(\sigma_{A+i}\right)\right)^{l}-\left(\mu_{\Lambda_{N_{0}}}^{(k)}\left(\sigma_{A+i}\right)\right)^{l}\right)\right| \\
& \quad \leqq 2\left(\frac{1}{\left|\Lambda_{N_{0}}\right|} \sum_{i: A+i \subset A_{N_{0}}^{(k)}} \mu_{A\left(N+N_{0}\right)}\left(\mu_{\Lambda_{N_{0}}}^{\tilde{\sigma}(k)}\left(\sigma_{A+i}\right)-\mu_{\left.A_{N_{0}}^{(k)}\left(\sigma_{A+i}\right)\right)^{2}}\right)^{1 / 2},\right.
\end{aligned}
$$

where $\mu_{\Lambda_{N_{0}}}^{\tilde{\sigma}_{(k)}}(\cdot)$ denotes the conditional expectation with respect to $\sum_{\left(\Lambda_{N_{0}}^{(k)}\right)^{c}}$ associated with the measure $\mu_{\Lambda\left(N+N_{0}\right)}$. The considerations in the proofs of Proposition 5.1 and of Lemma 5.2 show that

$$
(5.30) \leqq 2\left(\frac{1}{\left|\Lambda_{N_{0}}\right|} \sum_{i \in \Lambda_{N_{0}}} \sum_{j \in\left(\Lambda_{N_{0}}\right)^{c}} G_{i j}^{2}\right)^{1 / 2} .
$$

Therefore, setting

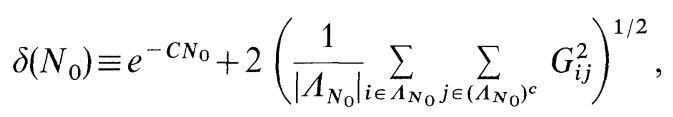

we get that

$$
\begin{aligned}
& \mid \frac{1}{\left|\Lambda\left(N+N_{0}\right)\right|} \sum_{i: A+i C \Lambda\left(N+N_{0}\right)}\left(\mu_{A\left(N+N_{0}\right)}\left(\sigma_{A+i}\right)\right)^{l} \\
& \quad-\frac{1}{L^{d N}} \sum_{k=1, \ldots, L^{d N}}\left(\frac{1}{\left|\Lambda_{N_{0}}\right|} \sum_{i: A+i \subset \Lambda_{N_{0}}^{(k)}}\left(\mu_{\Lambda_{N_{0}}^{(k)}}^{(k)}\left(\sigma_{A+i}\right)\right)^{l}\right) \mid \leqq \delta\left(N_{0}\right) .
\end{aligned}
$$

Since, for a Fisher base $\mathscr{F}_{\text {exp }}$, we have that $\delta\left(N_{0}\right) \rightarrow 0$, as $N_{0} \rightarrow \infty$, the law of large numbers permits us to conclude that the limit

$$
\begin{aligned}
& \lim _{\mathscr{F}_{\exp }} \frac{1}{\left|\Lambda_{N}\right|} \sum_{i: A+i \subset \Lambda_{N}}\left(\mu_{\Lambda_{N}}\left(\sigma_{A+i}\right)\right)^{l} \\
& \quad=\lim _{\mathscr{F}_{\exp }} \lim _{N \rightarrow \infty} \frac{1}{L^{d N}} \sum_{k=1, \ldots, L^{d N}}\left(\frac{1}{\left|\Lambda_{0}\right|} \sum_{i: A+i \subset \Lambda_{0}^{(k)}}\left(\mu_{\Lambda_{0}^{(k)}}\left(\sigma_{A+i}\right)\right)^{l}\right)
\end{aligned}
$$


exists and is independent of $n \in \mathbb{I}$ and $h \in \mathbb{l}$ h, $E \otimes \varrho$-a.e. By some simple arguments one can show that our statement remains true for the more general Fisher sequences $\mathscr{F}_{0}$ (and, in fact, that the limit is independent of $\mathscr{F}_{0}$ ). This ends the proof of Proposition 5.4.

\section{Appendix 1: Examples of Interactions}

Example 1: $(d=1)$. Let

$$
\hat{G}(q)=\frac{2 \pi}{2 q_{0}} \chi\left(|q| \leqq q_{0}\right), \text { with } \quad 0<q_{0}<\pi .
$$

Then

$$
G_{i j}=\frac{\sin q_{0}(i-j)}{q_{0}(i-j)},
$$

and application of the Poisson summation formula shows that

$$
\sum_{j \neq 0} \frac{\sin q_{0} j}{q_{0} j}=\frac{1}{2 q_{0}}-\frac{1}{2 \pi} .
$$

Therefore, the translation-invariant interaction given by $\hat{G}$ in (A.1) is weakly ferromagnetic.

Example 2: $(d=3)$. Let

$$
J(x)=\left(\frac{\sin q_{0}|x|}{q_{0}|x|}\right)^{3} .
$$

By explicit calculations we get

$$
\begin{aligned}
& (2 \pi)^{3} \widehat{J}(q) \equiv \int d_{3} x e^{-i q x} J(x) \\
& =\left\{\begin{array}{lll}
\frac{\pi^{2}}{2\left|q_{0}\right|^{3}} & \text { for } & |q|<\left|q_{0}\right| \\
-\frac{\pi^{2}}{4\left|q_{0}\right|^{3}}\left[\frac{3\left|q_{0}\right|-|q|}{|q|}\right] & \text { for } & \left|q_{0}\right| \leqq|q| \leqq 3\left|q_{0}\right| \\
0 & \text { for } & |q|>3\left|q_{0}\right| .
\end{array}\right.
\end{aligned}
$$

Formula (A.5) shows that $J \in J_{0}$, since

$$
\sum_{j \in \mathbb{Z}^{3} \backslash\{0\}} J_{0 j}=\frac{1}{(2 \pi)^{3}}\left[\frac{\pi^{2}}{2\left|q_{0}\right|^{3}}-1\right],
$$

for $0<\left|q_{0}\right|<\pi$, the interaction (A.4) is weakly ferromagnetic if $\left|q_{0}\right|<\left(\frac{\pi^{2}}{2}\right)^{1 / 3}$.

Example 3. (RKKY, $d=3$ ). First we note that for $d=3$ we have that

$$
\begin{aligned}
g(|x|) & \equiv \frac{1}{q_{0}^{3}} \int d_{3} q \chi\left(|q|<\left|q_{0}\right|\right) e^{i q x} \\
& =\frac{-\left|q_{0}\right||x| \cos \left|q_{0}\right||x|+\sin \left|q_{0}\right||x|}{\left(\left|q_{0}\right||x|\right)^{3}}=1 / 3+O\left(\left|q_{0}\right||x|\right)
\end{aligned}
$$


for small $|x|$, and $g \in W_{2,2}\left(\mathbb{R}^{3}\right)$. Note also that, for $f \in \mathscr{C}^{2}(\mathbb{R})$,

$$
\Delta f(|x|)=f^{\prime \prime}(|x|)+f^{\prime}(|x|) \frac{d-1}{|x|} .
$$

Using (A.7) and (A.8) one can show that the function

$$
J(|x|) \equiv \frac{1}{|x|+1}\left(\frac{-\left|q_{0}\right||x| \cos \left|q_{0}\right||x|+\sin \left|q_{0}\right||x|}{\left(\left|q_{0}\right||x|\right)^{3}}\right)
$$

belongs to $W_{2,2}\left(\mathbb{R}^{3}\right)$ and therefore, for $d=3$, its Fourier transform $\widehat{J}(q)$ is in $L_{1}\left(\mathbb{R}^{3}\right)$. Since

$$
\frac{1}{|x|+1}=\int_{0}^{\infty} d m e^{-m} e^{-m|x|}
$$

and

$$
\mathscr{F}\left(e^{-m|x|}\right)(q)=\frac{2 m}{\left(m^{2}+|q|^{2}\right)^{2}},
$$

we also see, using (A.7), that $J(|x|)$ is positive definite. We only have to show that the function

$$
\hat{G}(q) \equiv \sum_{l \in \mathbb{Z}^{3}} \hat{J}(q+2 \pi l), \quad q \in(-\pi, \pi)
$$

fulfills

$$
\|\widehat{G}\|_{\infty}<\infty \text {. }
$$

This follows from the monotonicity of the function $\widehat{J}(|q|)$.

Acknowledgements. We thank the Fonds National Suisse for financial support which made our collaboration possible.

\section{References}

1. Ruderman, M.A., Kittel, C.: Indirect exchange coupling of nuclear magnetic moments by conduction electrons. Phys. Rev. 96, 99 (1954)

2. Griffiths, R.B.: Nonanalytic behaviour above the critical point in a random Ising ferromagnet. Phys. Rev. Lett. 23, 17 (1969)

3. Kac, M.: On the partition function of a one-dimensional gas. Phys. Fluids 2, 8-12 (1959) Siegert, A.J.F.: Partition functions as averages of functionals of Gaussian random functions. Physica 26, 30-35 (1960)

4. Brascamp, H.J., Lieb, E.H.: On extensions of the Brun-Minkowski and Prékopa-Leindler theorem, including inequalities for log-concave functions and with applications to the diffusion equations. J. Funct. Anal. 22, 366-389 (1976)

5. Fortuin, C., Kasteleyn, P., Ginibre, J.: Correlation inequalities on some partially ordered sets. Commun. Math. Phys. 22, 89-103 (1971);

Battle, G.A., Rosen, L.: The FKG inequality for the Yukawa quantum field theory. J. Stat. Phys. 22, 123-192 (1980)

6. Aizenman, M., Lebowitz, J.L., Ruelle, D.: Some rigorous results on the SherringtonKirkpatrick spin glass model. Commun. Math. Phys. 112, 3-20 (1987) 
7. Fröhlich, J., Zegarlinski, B.: Some comments on the Sherrington-Kirkpatrick model of spin glasses. Commun. Math. Phys. 112, 553-566 (1987)

8. Ogielsky, A., Morgenstern, I.: Phys. Rev. Letts. 54, 428 (1985)

9. Sourlas, N.: Preprint ENS, Paris 1988

10. Bray, A.J., Moore, M.A.: Critical behaviour of the three dimensional Ising spin glass. Phys. Rev. B 31, 631 (1985); Lower critical dimension of Ising spin glasses: a numerical study. J. Phys. C 17, L463 (1984);

Bovier, A., Fröhlich, J.: A heuristic theory of the spin glass phase. J. Stat. Phys. 44, 347-391 (1986)

Fisher, D., Huse, D.: Phys. Rev. Lett. 56, 1601 (1986); J. Phys. A 20, L997 and L1005 (1987)

11. Fröhlich, J.: Mathematical aspects of the physics of disordered systems, pp. 725-893. In: Critical phenomena, random systems, gauge theories. Part II, Les Houches 1984. Osterwalder, K., Stora, R. (eds.). Amsterdam: North-Holland 1986

12. Fröhlich, J., Spencer, T.: On the statistical mechanics of classical Coulomb and dipole gases. J. Stat. Phys. 24, 617-701 (1981)

13. Griffiths, R.B.: Free energy of interacting magnetic dipoles. Phys. Rev. 176, 655-659 (1968); van Enter, A.C.D., van Hemmen, J.L.: The thermodynamic limit for long-range random systems. J. Stat. Phys. 32, 141-152 (1983)

14. Fontaine, J.-R.: Bounds on the decay of correlations for $\lambda(\nabla \phi)^{4}$ models. Commun. Math. Phys. 87, 385-394 (1982)

Communicated by T. Spencer

Received August 23, 1988 